\title{
Etude expérimentale de la fiabilité d'un module de puissance
}

\section{Experimental study of the reliability of a power module}

\author{
Philippe Pougnet $^{1,2}$, Gérard Coquery ${ }^{2}$ \\ ${ }^{1}$ Valeo Siemens eAutomotive, Cergy, philippe.pougnet.jv@valeo-siemens.com \\ ${ }^{2}$ VEDECOM, Versailles, gerard.coquery@ifsttar.fr
}

\begin{abstract}
RÉSUMÉ. En utilisation, les modules de puissance des véhicules électriques sont soumis à de très durs chargements thermiques. Les contraintes thermomécaniques qui en résultent impactent leur durée de vie. Comme les conditions spécifiques de montage et de refroidissement ont un effet significatif sur la fiabilité, il est important de tester les modules de puissance dans leurs conditions d'emploi et de vérifier qu'ils fonctionnent correctement pendant les durées des périodes de garantie qui peuvent s'étendre jusqu'à 15 ans. Pour provoquer des défaillances significatives de l'usure et du vieillissement en exploitation on soumet ces modules de puissance à des cycles répétés de contraintes dont les niveaux sont susceptibles d'être rencontrés en opération dans les conditions pire cas. Pour quantifier le niveau de dégradation, on suit l'évolution du vieillissement en mesurant des paramètres électriques et thermiques (tension émetteur- collecteur $V_{c e}$, résistance thermique $R_{t h}$ ). Les essais permettant d'évaluer la durée de vie des différentes technologies de modules de puissance sont basés sur l'application répétée de cycles de puissance.

ABSTRACT. In use, electric vehicle power modules are submitted to harsh thermal loads. The resulting thermo-mechanical stresses have an effect on their lifetime. As specific mounting and cooling conditions have a strong impact on reliability, it is important to test power modules in their specific use conditions and verify that lifetime matches warranty duration requirements which may extend to 15 years. To provoke failures which are significant of application wear and aging, repeated stress cycles are applied to power modules. Stress levels correspond to worst case scenarios. To evaluate the degradation level, aging is characterized measuring electric and thermal parameters (emettor- collector voltage $V_{\text {ce }}$, thermal resistance $R_{t h}$ ). The test leading to lifetime assessment of various power module technologies are based on applying power cycles.

MOTS-CLÉS. Fiabilité, Module de puissance, Test de cyclage en puissance (PCT), Test de tenue en cycles thermiques (TCT). KEYWORDS. Reliability, Power Module, Power Cycling Test (PCT), Thermal Cycling Testing (TCT).
\end{abstract}

\section{Introduction}

Pour pouvoir mettrre sur le marché des véhicules automobiles hybrides et électriques accessibles au plus grand nombre, il est nécessaire de cévelopper à coût de fabrication optimal des convertisseurs de puissance intégrés de type continu -alternatif (DC-AC), continu-continu (DC-DC) ou alternatif-continu (AC-DC). L'utilisation de modules de puissance compacts permet d'optimiser le coût des convertisseurs embarqués et de garantir leurs performances en terme de rendement énergétique, de volume et de masse.

Les circuits électroniques des modules de puissance sont réalisés en attachant des composants électroniques sous forme de puces nues sur des substrats qui sont à la fois isolants électriques et conducteurs thermiques. Les architectures des modules de puissance sont très variées et sont en constante évolution. Avant de lancer une fabrication en série de véhicules utilisant des modules de puissance, il est recommandé .de vérifier qu'ils fonctionneront correctement dans les conditions d'usage prévues. Lorsque les puces ou les technologies d'assemblage sont nouvelles on ne dispose pas de suffisamment de connaissances sur la physique de la défaillance pour effectuer des prédictions de durée de vie. On évalue alors leur durée de vie par des essais accélérés de vieillissement. Cette approche expérimentale permet de connaître les modes et les mécanismes de défaillance provoqués par les contraintes opérationnelles et environnementales. Sur la base des résultats obtenus, cette approche permet de proposer des améliorations de conception ou de procédés. 


\section{L’approche expérimentale}

\subsection{Les chargements d'usage}

Les températures de transistors IGBT et de diodes de puissance ont été mesurées sur le module de puissance d'un véhicule électrique lors d'un fonctionnement réel sur un parcours urbain et périurbain [COQ 01]. Ces mesures (Figure 1)mettent en évidence que les températures de jonction des composants de puissance varient de manière cyclique. Les variations de température de jonction sont de l'ordre de $50^{\circ} \mathrm{C}$. Les températures de jonction peuvent grimper juqu'à $120^{\circ} \mathrm{C}$.

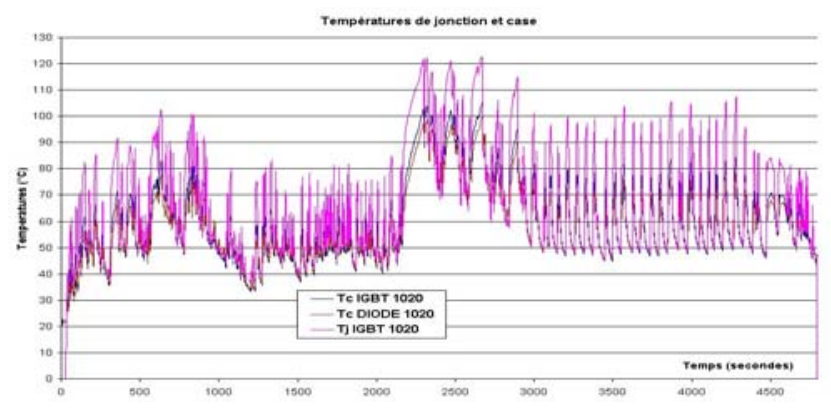

Figure 2.1. Température de jonction et de boîtier du transistor IGBT et de la diode de puissance en fonction du temps de trajet

\subsection{L'impact des conditions de montage des modules de puissance sur leur durée de vie}

La figure 2 représente les différentes couches de l'empilement d'un module de conception classique (conduction thermique vers la semelle du module et évacuation de la chaleur par circulation d'un liquide de refroidissement).

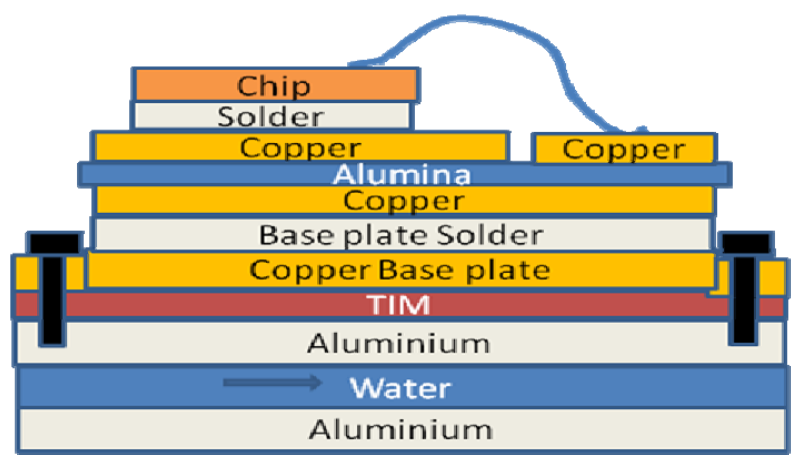

Figure 2.2. Empilement d'un module de puissance à refroidissement par la semelle

\subsection{Les essais de cyclage permettant d'évaluer la durée de vie}

Les technologies des modules de puissance sont en évolution permanente. Acquérir une connaissance précise des modes de vieillissement offre au concepteur de système embarqué les données pertinentes pour le montage thermomécanique choisi, la stratégie de maintenance et l'aide à la décision sur le niveau de confiance de la garantie requise. Cette approche de la fiabilité est basée sur des essais accélérés spécifiques comme le TCT (Thermal Cycling Test) qui révèle l'impact strict des conditions climatiques et des cycles lents de température occasionnés par les trajets et le PCT (Power Cycling Test) qui établit les modes de défaillance liés aux cycles de traction-freinage. Dans ce papier sont abordés les essais expérimentaux PCT. Cette approche expérimentale se distingue de l'approche déterministe de la consommation de durée de vie [COI 12] qui est basée sur la modélisation des pertes thermiques générées par les scenarios du profil de mission et sur les lois de la physique de la défaillance. 


\section{Essais expérimentaux de tenue en cycles de puissance}

\subsection{Objectif et principe du PCT}

Le test de vieillissement cyclique PCT a pour objectif d'évaluer le vieillissement des transistors et diodes d'un module de puissance en les exposant à des cycles de puissance représentatifs en nombre et en amplitude des cycles appliqués sur le produit dans les conditions d'emploi. Le principe du test PCT consiste à appliquer sur les composants sous test des impulsions de puissance de manière cyclique (Figure 3) et à suivre l'évolution de la température de jonction en mesurant avec un courant constant de très faible amplitude la chute de tension aux bornes du transistor ou de la diode [LUT 11].

Typiquement, la durée de l'impulsion de courant de puissance (ton) est de 10 secondes. La durée du refroidissement ( $\mathrm{t}$ off) du module avant l'application d'un nouveau cycle est de 20 secondes. La mesure indirecte de la température de jonction avant et après l'application d'une impulsion de courant de puissance permet de connaître la variation de la température de jonction $(\mathrm{Tj})$ du composant sous test. Conjuguée à la mesure de l'amplitude du courant, elle conduit à la mesure de la puissance. Pour un transistor IGBT on obtient la température équivalente de jonction en caractérisant au préalable la chute de tension entre le collecteur et l'émetteur (Vce) en fonction de la température. Pour une diode de puissance, on obtient la température de jonction en caractérisant la chute de tension directe en fonction de la température.

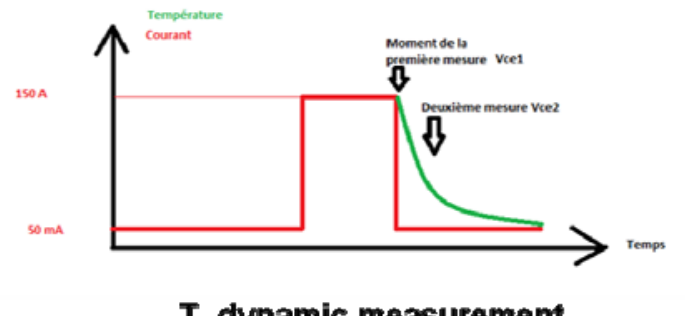

$T_{\jmath}$ dynamic measurement

Figure 3.1. Principe du test de cyclage en puissance (PCT)

\subsection{Exemple d'essais PCT}

Trois modules de puissance sont testés simultanément. Ils sont répertoriés par les lettres A, B et C. L'architecture de ces modules est de type refroidissement par la semelle (Figure 2). Des thermocouples sont placés sous les puces actives au niveau de la semelle du module. Le dépôt du matériau d'interface thermique est réalisé par sérigraphie. Les modules sous test sont fixés sur la plaque de refroidissement de la même manière que sur véhicule. La température du liquide de refroidissement est fixée à $45^{\circ} \mathrm{C}$. L'amplitude du courant de puissance est ajustée afin que la température de jonction à la fin de l'impulsion n'excède pas $125^{\circ} \mathrm{C}$. Le courant de mesure de Vce est fixé à $100 \mathrm{~mA}$.

Avant d'effectuer les essais, des caractérisations mécaniques tridimensionnelles de la semelle sont faites ainsi que des caractérisations de Vce ou de $\mathrm{Vd}$ en fonction de la température et des caractérisations par microscopie acoustique des différentes interfaces des couches des modules.

\subsection{Résultats}

Les donnés sauveagardées pendant le test PCT permettent de connaître pour chaque cycle la contrainte appliquée (amplitude de la variation de température de jonction) et de suivre le vieillissement (température de jonction, Iges).

La figure 4 représente l'évolution de la variation de la température de jonction mesurée lors de l'activation des cycles de puissance. La variation de la température de jonction fluctue autour d'une valeur centrale qui est de $83^{\circ} \mathrm{C}$ (figure 8 ). 


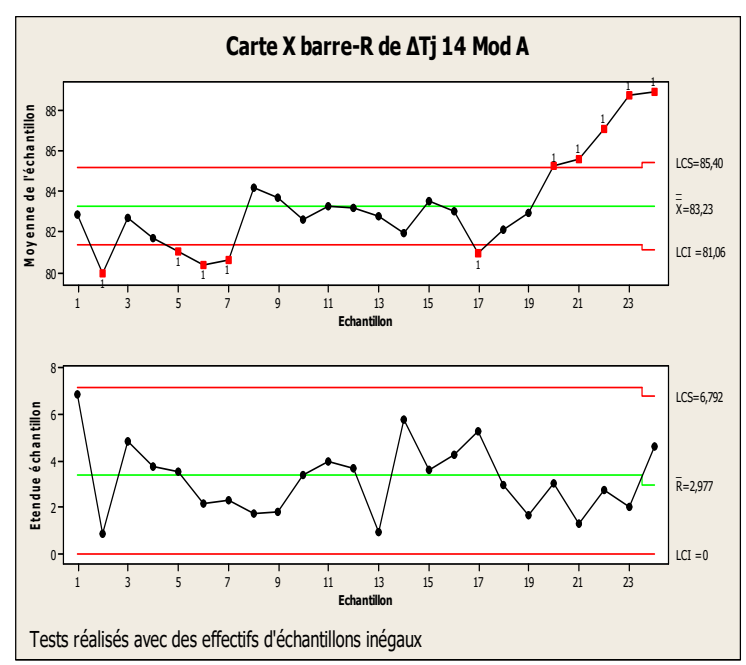

Figure 3.2. Variation de la température de jonction de la puce IGBT 14 en fonction du nombre de cycles de puissance

Au-delà de 180000 cycles, on constate une forte augmentation de la température de jonction Pour détecter avec précision le vieillissement, des mesures statiques sont réalisées de manière périodique (par exemple tous les 10000 cycles). Ces caractérisations consistent à arrêter les cycles de puissance et à appliquer des impulsions de puissance calibrées et à mesurer Vce. La figure 5 représente la variation relative de Vce en fonction des cycles de puissance. Le seuil critique de détection de défaillance est déterminé à 5\%. Il est atteint à 207000 cycles.

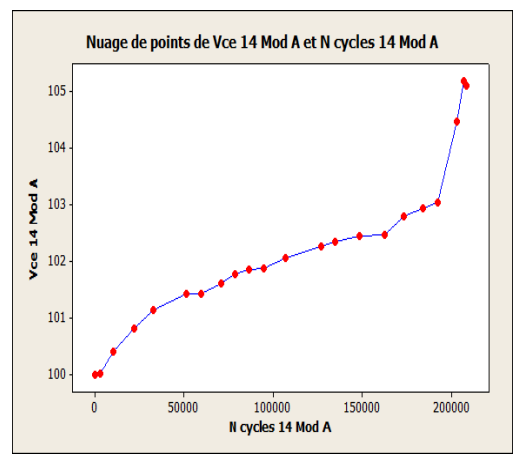

Figure 3.3. Variation de Vce de la puce IGBT 14 en fonction du nombre de cycles de puissance

\section{Discussion}

On observe sur les trois modules testés que la variation relative de Vce dépasse le seuil critique de $5 \%$ sur quatre puces IGBT. Ce dépassement de seuil indique une défaillance de la connexion par fil de bonding.

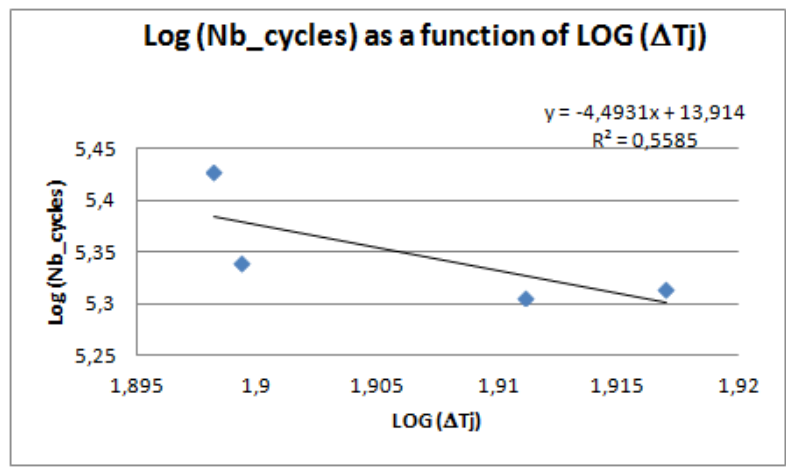

Figure 4.1. Nombre de cycles à défaillance en fonction de la variation moyenne de température de jonction 
La figure 6 représente en échelle logarithmique comment pour ces quatre défaillances le nombre de cycles jusqu'à défaillance varie avec la contrainte appliquée. Cette évolution y est décrite par une droite de tendance. On peut utiliser alors ce modèle pour estimer le nombre de cycles conduisant à défaillance pour les niveaux de contrainte plus faibles rencontrés en service (Figure 7). Pour une variation de température de jonction de $40^{\circ} \mathrm{C}$, le nombre de cycles de puissance provoquant une défaillance est de 4 millions.

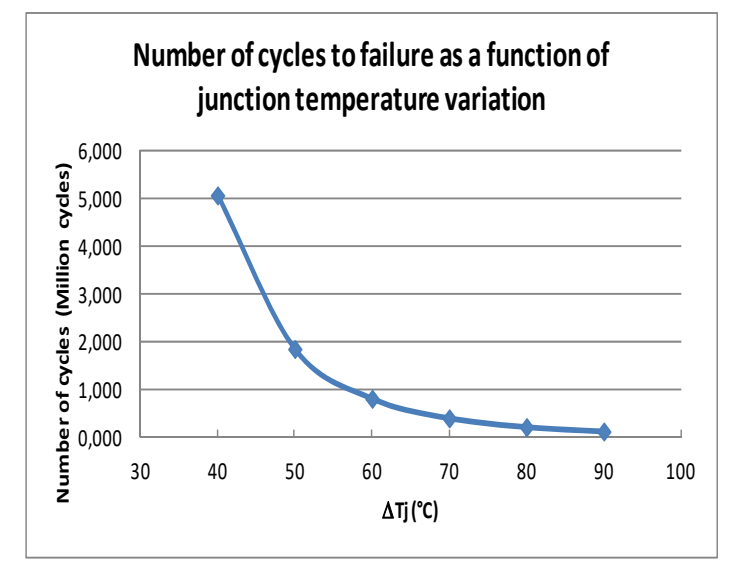

Figure 4.2. Prédiction du nombre de cycles à défaillance en fonction de la variation de température de jonction

\section{Conclusion}

Le succès industriel des véhicules électrifiés (hybrides et électriques) repose pour une part sur le développement de modules de puissance à la fois compacts, performants à coût réduit. Les technologies de réalisation des modules de puissance évoluent rapidement. Les innovations introduites dans les composants de puissance, les matériaux d'attache et de connexion ou les architectures peuvent être caractérisées par une approche expérimentale utilisant des essais accélérés de référence. Ces essais sont basés sur les profils de mission des modules et sur leurs conditions spécifiques de montage mécanique et de refroidissement. Les essais de tenue en cycles de puissance (PCT) reproduisent les contraintes appliquées par les activations de modules et permettent d'identifier et de comprendre les mécanismes de défaillance. Pour une technologie donnée, le PCT permet d'évaluer la durée de vie ainsi que la variabilité de cette durée de vie au sein d'un module et de module à module. En l'absence d'outils de simulation fiable, cette approche expérimentale est la seule à ce jour qui permet de valider en durée de vie et garantie d'usage une innovation dans le domaine des convertisseurs de puissance des véhicules électriques.

\section{Bibliographie}

[COI 12] CoÏA-Huret N., Merabet B., Thionville A., "Estimation de la Fiabilité de Modules de puissance IGBT en phase de conception Journée SIA 15/11/2012 "La Fiabilité et la Robustesse par la Simulation numérique.

[COQ 01] Coquery G., CARUbelli S.N., Lallemand J.P., LeCoCQ R., LhOtellier F., De Viry D., Dupuy Ph V., « Power module lifetime estimation from chip temperature direct measurement in an automotive traction inverter », ESREF 2001.pp. 1695-1700

[LUT 11] Lutz J., Schlangenotto H., Scheuermann U., De Doncker R., Semiconductor Power Devices ISBN 978364211242 ISTE Springer Editions, 2011. 